\title{
THE EFFICIENCY OF THE FIGHT AGAINST HIV, IN CONSTANTA, FROM THE PERSPECTIVE OF MEUROLOGICAL COMPLICATIONS
}

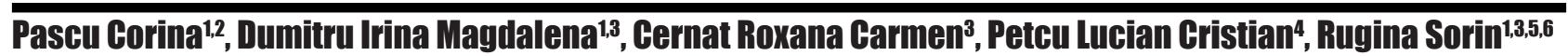

\author{
${ }^{1}$ Doctoral School of Medicine, Ovidius University of Constanta \\ ${ }^{2}$ Department of Neurology, County Clinical Emergency Hospital of Constanta \\ ${ }^{3}$ Clinical Infectious Diseases Hospital of Constanta \\ ${ }^{4}$ Faculty of dentistry, Ovidius University of Constanta \\ ${ }^{5}$ Romanian Academy of Medical Sciences \\ ${ }^{6}$ Academy of Romanian Scientists
}

Lucian Cristian Petcu

email: crilucpetcu@gmail.com phone: +40726251948

\begin{abstract}
In order to control and end the AIDS epidemic, we all need to hit the 0-0-0 target. That means 0 HIV infections, 0 mortality caused by AIDS and 0 discrimination. We performed a retrospective observational analytical study on 166 HIV positive patients with a neurological complication, diagnosed between June 2012 and June 2020, in Clinical Infectious Diseases Hospital of Constanta. We collected data from the observational charts that revealed if the patient was known with HIV infection at the time of the neurological complication diagnosis, information about the treatment (how many treatment regimens, classes of antiretroviral drugs, CNS Penetration-Effectiveness Rank), treatment compliance. The patients were divided into two groups: first group "opportunistic infections (OI)" and second group "other neurological conditions (ONC)". 16 patients (9,6\%) have the neurological condition as first manifestation of HIV infection. Opportunistic infections, correlated with a poor immunological status continue to have a high percentage $(46,98 \%)$ of neurological complications, being associated with a poor treatment compliance $(p<0,001)$. Integrase inhibitors were associated, in this study, more with opportunistic infections and less with other neurological conditions $(O R=2,885 ; 95 \%$ IC), this finding needs further research. Long term survivors have problems with treatment compliance manifesting treatment fatigue, even if they have treatment regimens with good CNS penetrability. Poor compliance is associated with high risk of opportunistic infections and opportunistic infections have a higher mortality than other neurological complications. Our task for the future is to find ways to improve treatment adherence in order for HIV patients to have a better and longer life.
\end{abstract}

Keywords: HIV, neurological complication, compliance, integrase inhibitors, mortality

\section{Introduction}

The implementation of combined antiretroviral therapy (cART) across the world increased survival of patients but also changed the neurological complications of HIV infections spectrum, by reducing the incidence of opportunistic infections (OI) and adding neurodegenerative and neurovascular diseases.

One of the major hurdles of complete eradication of HIV in the era of cART is the presence of sanctuaries and reservoires of replications competent HIV virions. One of this sites is central nervous system (CNS) (1). It is believed that, if antiretroviral drugs could penetrate the blood-brain barrier, this reservoir could be suppressed. In order to estimate the CNS exposure to antiretroviral drugs the reserchers developed the CNS penetration effectiveness score (2). If the score is high than the viral load in cerebrospinal fluid should be low $(3,4)$.

cART allows the optimization of CNS penetration effectiveness score but the neurological complications of HIV remain an important issue. One of the risk factors for the persistence of neurological complications, especially, opportunistic infections is poor treatment compliance. Poor adherence to treatment meaning high mortality. 


\section{Materials and methods}

166 HIV positive patients diagnosed with a neurological complication were enrolled in the study between June 2012 and June 2020. The data was collected from the observational charts from Clinical Infectious Diseases Hospital of Constanta.

The experimental data were processed with IBM SPSS statistics 23 software. The procedures used were: descriptive statistics, graphs, parametric statistical tests, nonparametric statistical tests for categorical data ( $\chi 2$ association test, with OR calculation, $\chi 2$ test for comparison of two proportions) and nonparametric statistical tests for ordinal data (Mann-Whitney U test, Kruskal-Wallis test).

The patients were divided into two groups: first group "opportunistic infections (OI)" and second group "other neurological conditions (ONC)". In this study, the term "OI" included: Progressive Multifocal Leukoencephalopathy (PML), CNS Toxoplasmosis (CT), CMV encephalitis (E.CMV), Herpes simplex encephalitis (E.HVS1,2), CNS cryptococcosis (CrSNC), Neurocysticercosis (NCC), Central nervous system tuberculosis (TBC SNC), Primary cerebral lymphoma (LPC). In the second group were the patients with HIV associated dementia (HAD), stroke (AVC), peripheral nervous system diseases (HIV SNP) (Figure 1). We gathered data about the status of the patient (HIV positive in medical history or neurological disease as first manifestation of HIV), and about the treatment -how many treatment regimens, classes of antiretroviral drugs, CNS Penetration-Effectiveness Rank.

\section{Results}

The distribution of the neurological complications found in our study are detailed in Figure 1.

In the first group, OI, the neurologic condition was the first manifestation of HIV for 8 patients $(10,3 \%)$, out of 78 patients. In the second group, ONC, $8(9,1 \%)$ out of 80 patients were in the same situation. No statistically significant differences were found between the proportion of patients with OI as first manifestation of HIV and the proportion of patient with ONC as first sign of HIV ( $p>0,05)$.

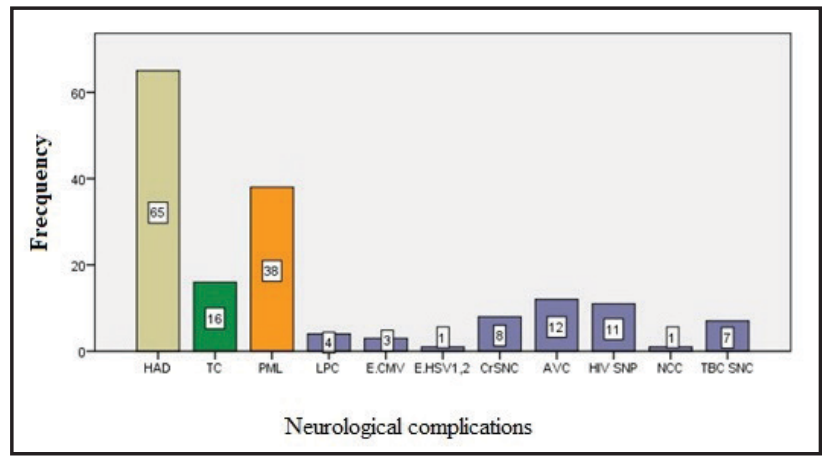

Figure 1. Neurological complications of HIV infection ( $H A D=H I V$ associated dementia, $C T=$ CNS Toxoplasmosis, $P M L=$ Progressive Multifocal

Leukoencephalopathy, $L P C=$ Primary cerebral lymphoma, E.CMV = CMV encephalitis, E.HVS1,2= Herpes simplex encephalitis, $C r S N C=C N S$ cryptococcosis, $A V C=$ stroke, $H I V S N P=$ peripheral nervous system diseases, $N C C=$ Neurocysticercosis, $T B C$

$\mathrm{SNC}=$ Central nervous system tuberculosis)

From a total of 166 patients, $138(83,1 \%)$ were under combined antiretroviral therapy (cART). There is no association between the two variables "Group" and "cART": $\chi 2$ calc $=0.064$, df $=1, p=0.800>\alpha=0.05$. The risk to find patients under cART with OI is equal with the risk to find patients under cART with ONC: OR (odds ratio) $=1.143 ; 95 \% \mathrm{IC}=(0.408,3.205)$.

Both group of patients have had many treatment regimens through years as seen in Table 1, with a median of 3 for OI and 2,5 for ONC.

Table 1 Number of treatment regimens

\begin{tabular}{|l|c|c|}
\hline \multirow{2}{*}{} & \multicolumn{2}{|c|}{ Group } \\
\cline { 2 - 3 } & $\begin{array}{c}\text { Opportunistic } \\
\text { infections }\end{array}$ & $\begin{array}{c}\text { Other neurological } \\
\text { conditions }\end{array}$ \\
\hline $\mathrm{N}$ & 78 & 88 \\
\hline Mean & 2.76 & 2.73 \\
\hline Median & 3.00 & 2.50 \\
\hline Mode & 3.00 & 2.00 \\
\hline Std. Deviation & 2.03 & 1.61 \\
\hline Minimum & .00 & .00 \\
\hline Maximum & 8.00 & 6.00 \\
\hline
\end{tabular}

The study focuses next on the correlation between $\mathrm{OI}$ and $\mathrm{ONC}$ and the classes of antiretroviral drugs. The risk to find patients 
under nucleoside reverse transcriptase inhibitors (NRTIs) with OI is equal with the risk to find patients under NRTI with ONC: OR (odds ratio) $=0.983 ; 95 \% \mathrm{IC}=(0.337,2.559)$.

We observed similar findings with the other classes of antiretroviral drugs - non-nucleoside reverse transcriptase inhibitors, protease inhibitors, fusion inhibitors, CCR5 antagonists, cd4 attachment inhibitors, boosting agents - with the exception of integrase inhibitors (INSTI).

"Group" and "INSTI" are dependent variables: $\chi 2$ calc $=7.925, \mathrm{df}=1, \mathrm{p}=0.005<\alpha$ $=0.05$.

The risk to find patients under INSTI with $\mathrm{OI}$ is 2.885 higher than the risk to find patients under INSTI with ONC: OR (odds ratio) $=2.885$; $95 \% \mathrm{IC}=(1.357,6.130)$ (Figure 2$)$.

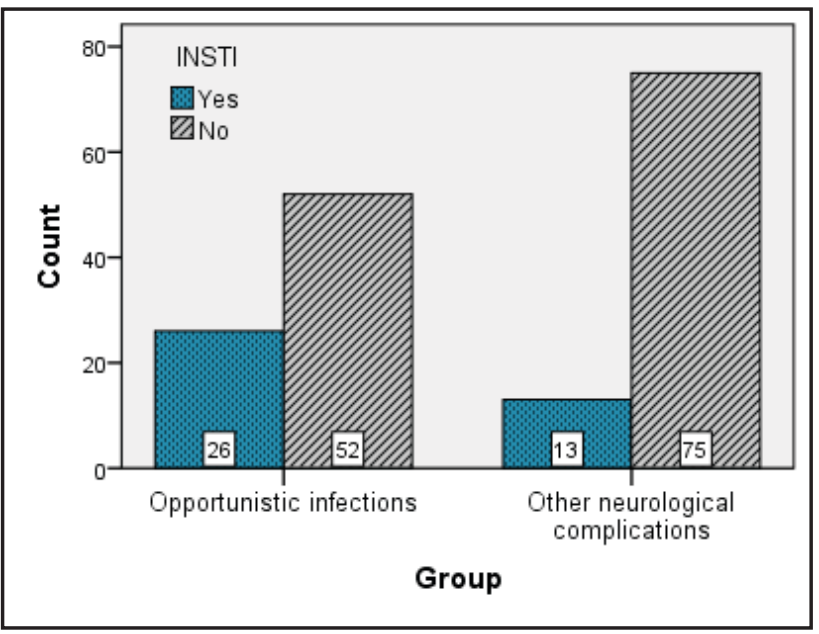

Figure 2. The distribution of patients under INSTI in the two groups: OI and ONC

The mean and median values of the CNS penetration effectiveness (CPE) scores, in the two groups, are high, even if all the patients have a neurological complication (Table 2).

Table 2 CNS penetration effectiveness score of cART

\begin{tabular}{|c|c|c|}
\hline & \multicolumn{2}{|c|}{ Group } \\
\hline & $\begin{array}{l}\text { Opportunistic } \\
\text { infections }\end{array}$ & $\begin{array}{l}\text { Other neurological } \\
\text { conditions }\end{array}$ \\
\hline $\mathrm{N}$ & 78 & 88 \\
\hline Mean & 8.13 & 7.55 \\
\hline Median & 8.50 & 8.00 \\
\hline Mode & 9.00 & 8.00 \\
\hline Std. Deviation & 3.10 & 2.66 \\
\hline Minimum & .00 & .00 \\
\hline Maximum & 14.00 & 13.00 \\
\hline
\end{tabular}

The distribution of CNS penetration effectiveness (CPE) score of cART has no statistically significant difference between OI and ONC ( $\mathrm{p}=0.120$, Mann-Whitney U Test).

$65(39,2 \%)$ patients from 166 patients with neurological complications of HIV infection, were compliant with the treatment, $90(54,2 \%)$ were not compliant and $11(6,6 \%)$ were naïve patients (Figure 3).

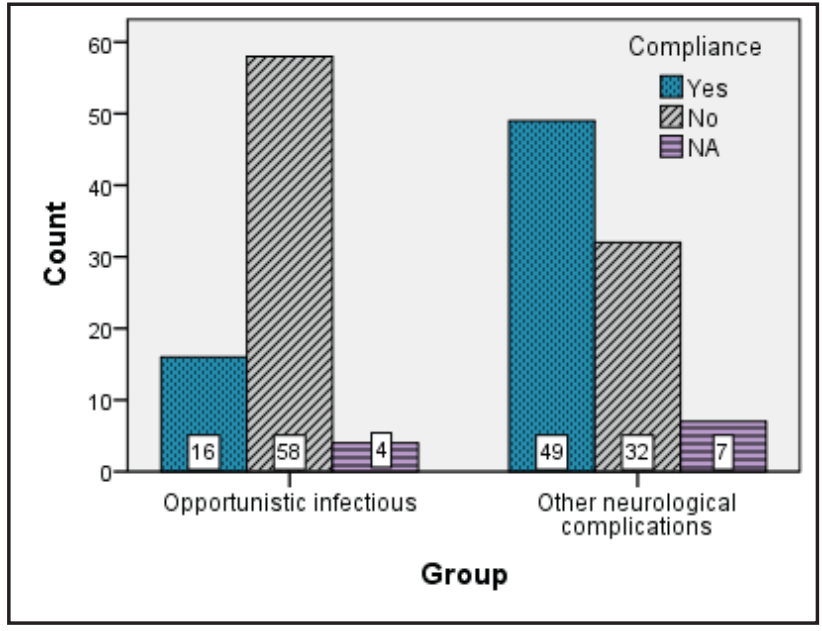

Figure 3 Compliance to treatment

Table 3 Mortality and survival of patients with neurological complications: OI and ONC

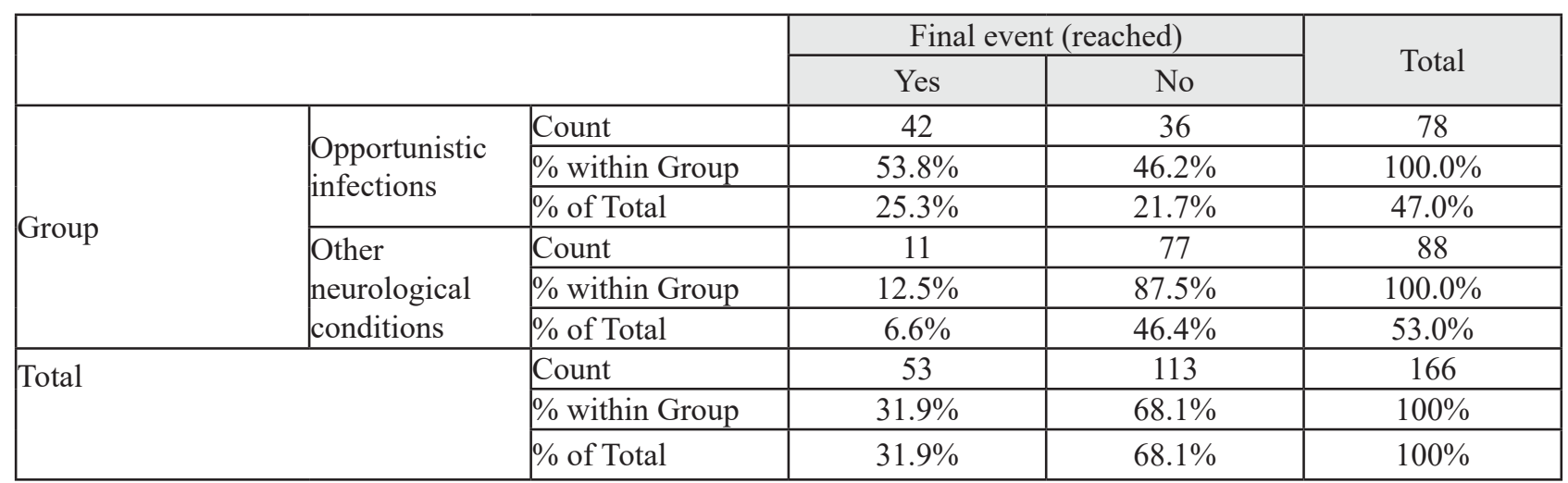


"Group" and "Compliance" are dependent variables: $\chi 2$ calc $=24.570, \mathrm{df}=2, \mathrm{p}<0.001<\alpha$ $=0.05$.

$42(53,8 \%)$ patients with OI and $11(12,5 \%)$ patients with ONC reached the final event-death (Table 3).

"Group" and "Final event" are dependent variables: $\chi 2$ calc $=32.524, \mathrm{df}=1, \mathrm{p}<0.001<$ $\alpha=0.05$.

\section{Discussion}

The percentage of $9,6 \%$ patients that have the first manifestation of HIV a neurological condition, is similar with the data from literature where the percentage found is $5-10 \%$. At autopsy the percentage in which the CNS is involved, is much higher reaching $90 \%(5,6)$.

In Romania, at the end of 2019, there were 16486 HIV positive patients and under cART 13437 (81,5\%) patients (7). In our study, 83,1\% of the patients were under cART, so a very good percentage considering that we only included the patients with a neurological complication of HIV. This shows a good accessibility of the treatment but also a persistence of the neurological complications of HIV, even under cART. In the first group, OI, the adherence to the treatment is an important subject, justifying the susceptibility of this patients to opportunistic infections. In the second group, ONC, we have to take into consideration other risk factors like metabolic and vascular factors that play an important role in the onset of cognitive decline, stroke, or polyneuropathy.

NRTIs are associated, mostly, with neuropathy. In our study, only $11(6,63 \%)$ patients had a peripheral nervous system condition, so not significant for the group.

The adverse effects of INSTIs are mostly psychiatric (8). This could influence treatment compliance and raise the risk of OI through treatment failure. But the correlation between INSTIs and OI needs further studies.

The patients with poor compliance are at higher risk to develop an OI.

The risk of death is statistically higher for the patients with OI.

\section{Conclusions}

Opportunistic infections account for $46,98 \%$ of neurological complications in this study. The rest is represented by HIV associated dementia, stroke and peripheral nervous system diseases.

The percentage of patients that have a neurological condition as first manifestation of $\mathrm{HIV}$ is $9,6 \%$ and considering that many of the neurological complications are associated with an advanced stage of the disease, we have to have this number as close to " 0 " as possible in order to have a good control of the disease.

In our center, we have good accessibility to treatment, but the problem of compliance still exists and generates complications. Compliance is associated strongly with the onset of opportunistic infections. So, we have to work to find better ways to improve adherence to HIV treatment.

We have to raise awareness for the "nonclassic" risk factors for neurologic complications like metabolic, vascular factors that play an important role in virally suppressed patients, by screening and treating for atherosclerosis, dyslipidemia, hypertension.

Integrase inhibitors were associated, in this study, more with opportunistic infections and less with other neurological conditions, and this needs further research.

The risk of death is statistically higher for the patients with opportunistic infections, so the fight for a better compliance, as an important risk factor for opportunistic infections must be a priority.

\section{Acknowledgments}

The authors declare that there is no conflict of interest.

\section{References}

1. Joseph J, Cinque P, Colosi D, Dravid A, Ene L, Fox H, et al. Highlights of the Global HIV1 CSF Escape Consortium Meeting, 9 June 2016, Bethesda, MD, USA. Vol. 2, Journal of virus eradication. 2016. p. 243-50.

2. Letendre SL, Ellis RJ, Ances BM, 
McCutchan JA. Neurologic complications of HIV disease and their treatment. Top HIV Med. 2010;18(2):45-55.

3. Letendre S, Marquie-Beck J, Capparelli E, Best B, Clifford D, CollierAC, etal. Validation of the CNS Penetration-Effectiveness rank for quantifying antiretroviral penetration into the central nervous system. Arch Neurol. 2008 Jan;65(1):65-70.

4. Marra CM, Zhao Y, Clifford DB, Letendre S, Evans S, Henry K, et al. Impact of combination antiretroviral therapy on cerebrospinal fluid HIV RNA and neurocognitive performance. AIDS. 2009 Jul;23(11):1359-66.

5. Bacellar H, Muñoz A, Miller EN, Cohen BA, Besley D, Selnes OA, et al. Temporal trends in the incidence of HIV-1-related neurologic diseases: Multicenter AIDS Cohort Study, 1985-1992. Neurology. 1994 Oct;44(10):1892-900.

6. Rachlis AR. (1998) Neurologic manifestations of HIV infection. Postgrad Med.;103:1-11.

7. Institutul de Boli Infectioase "Prof. Dr. Matei Bals" Compartimentul pentru monitorizarea si evaluarea infectiei HIV/SIDA in Romania. Evolutia infectiei HIV/SIDA in Romania 31 Decembrie 2019. http://www.cnlas.ro/com jce/date-statistice.html: Institutul de Boli Infectioase "Prof. Dr. Matei Bals", 2019.

8. Cohen C, Elion R, Ruane P, Shamblaw D, DeJesus E, Rashbaum B, et al. Randomized, phase 2 evaluation of two singletablet regimens elvitegravir/cobicistat/ emtricitabine/tenofovir disoproxil fumarate versus efavirenz/emtricitabine/tenofovir disoproxil fumarate for the initial treatment of HIV infection. AIDS. 2011 Mar;25(6):F7-12 